\title{
Evaluation of factors increasing the risk of silent brain infarction using multidetector computed tomography in patients undergoing carotid artery stenting
}

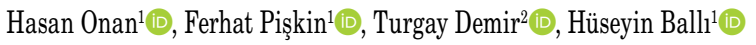 \\ ${ }^{1}$ Department of Radiology, Çukurova University Faculty of Medicine, Adana, Turkey \\ ${ }^{2}$ Department of Neurology, Çukurova University Faculty of Medicine, Adana, Turkey \\ Received: January 09, 2021 Accepted: February 22, 2021 Published online: March 29, 2021
}

\section{ABSTRACT}

Objectives: This study aims to identify the factors increasing the risk of silent brain infarction using multidetector computer tomography (MDCT) in patients undergoing carotid artery stenting (CAS).

Patients and methods: Medical data of a total of 24 patients (12 males, 12 females; mean age: $65.4 \pm 74$ years; range, 50 to 76 years) who underwent CAS between December 2017 and July 2019 were retrospectively analyzed. The plaque characteristics of carotid arteries and the aortic arch structures were determined using MDCT before the procedure. The patients were divided into two groups based on the diffusion-weighted images after the procedure. Those with and without silent brain infarction were in Group 1 ( $n=10)$ and Group 2 ( $n=14)$, respectively. Both groups were compared in terms of aortic arch structures and the plaque characteristics.

Results: There was a significant difference between the groups in terms of plaque in the aortic arch ( $n=8$ vs. $n=5$, respectively, $p=0.047$ ). The number of irregular plaque surfaces, plaque length, and plaque volume were significantly higher in Group 1 than Group 2 ( 8 vs. 5 , respectively; $\mathrm{p}=0.047 ; 21.8$ vs. 17.6 , respectively; $\mathrm{p}=0.045$, and 991.1 vs. 740.3 , respectively; $\mathrm{p}=0.015$ ). The percentages of lipid components in total plaque volume were significantly higher in Group 1 ( $\mathrm{p}=0.026)$. The receiver operating characteristic analysis revealed that $17.8 \%$ of lipid components in total volume had $64.3 \%$ sensitivity and $90 \%$ specificity in the differentiation of the groups.

Conclusion: Evaluation of aortic arch structures and the plaque characteristics in carotid arteries using MDCT may be useful for predicting the risk of silent brain infarction after CAS.

Keywords: Aortic arch, carotid stenting, computed tomography, plaque.

Carotid artery stenting (CAS) has been widely used to treat patients with carotid artery stenosis since the 2000s. ${ }^{[1]}$ The technical success rates of CAS have been reported to be similar to carotid endarterectomy (CEA); however, the risk of silent brain infarction (SBI) in the post-procedural period is higher in patients undergoing CAS. ${ }^{[1-3]}$ Silent brain infarction is defined as a vascular brain lesion of embolic origin. Although SBI is asymptomatic in the acute phase, it has a negative effect on cognitive functions in the long-term. Therefore, in CAS planning, patients at high risk of developing SBI should be detected prior to the procedure and referred for CEA treatment, instead of CAS. ${ }^{[3,4]}$ Although there is no clear consensus yet, a few studies have recently provided evidence that characteristics of the aortic arch and its branches may be associated with the development of ipsilateral SBI during the postprocedural period. Therefore, it may be important to identify the characteristics of the aortic arch and its branches in planning CAS. ${ }^{[5,6]}$

Recent technological advances have enabled the high-spatial resolution images to be obtained using multidetector computed tomography (MDCT). Thus, anatomical variation and pathological changes of vessels can be much better demonstrated with three-dimensional imaging. ${ }^{[7,8]}$ In addition, on postprocessing analysis, structure characteristics of

Corresponding author: Ferhat Pişkin, MD. Çukurova Üniversitesi Tip Fakültesi Radyoloji Anabilim Dalı, 01790 Sarıçam, Adana, Türkiye.

Tel: +90 555 - 7368283 e-mail: ferhatcpiskin@gmail.com

\section{Citation:}

Onan H, Pişkin F, Demir T, Ballı H. Evaluation of factors increasing the risk of silent brain infarction using multidetector computed tomography in patients undergoing carotid artery stenting. Cardiovasc Surg Int 2021;8(1):28-34. 
atheromatous plaque in vessels can reveal using specific software. ${ }^{[7,8]}$

In the present study, we aimed to analyze images obtained in MDCT before CAS and to identify differences in the vascular structures of patients having SBI after the procedure.

\section{PATIENTS AND METHODS}

This single-center, retrospective study was conducted at Çukurova University Faculty of Medicine, Department of Interventional Radiology between December 2017 and July 2019. Data of the patients who underwent CAS were analyzed retrospectively. Of a total of 108 patients, 24 (12 males, 12 females; mean age: $65.4 \pm 74$ years; range, 50 to 76 years) who met the inclusion criteria were recruited. Inclusion criterion was undergoing imaging of the carotid arteries using MDCT within the last month before CAS. Neurological and general physical examinations were performed by an experienced neurologist before and after procedure. Patients with newly developed neurological symptoms after the procedure were excluded from the study. The medical history of the patients was obtained from the institutional database. A written informed consent was obtained from each patient. The study protocol was approved by the Çukurova University Faculty of Medicine Clinical Research Ethics Committee (No: 09/2019-91). The study was conducted in accordance with the principles of the Declaration of Helsinki.

\section{MDCT image analysis}

All MDCT scans were performed on a 160-detector CT scanner (Toshiba Aquilion ${ }^{\mathrm{TM}}$ PRIME; Otawara, Japan). The scanning area was determined to cover the lower limit of the heart and cerebral cortex. Post-processing of the data was evaluated using a dedicated workstation (Vitrea ${ }^{\circledR}$; Vital Images Inc., MN, USA) by an experienced vascular radiologist.

The aortic arch was grouped according to anatomical variation and elongation (Figure 1). The aortic arch variation was defined according to the arch vessels' origin. The aortic arch elongation was defined according to the vertical distance from the origin of the innominate artery to the top of the arch (elongated aortic arch: $>1 \mathrm{~cm}$ in diameter). ${ }^{[9,10]}$

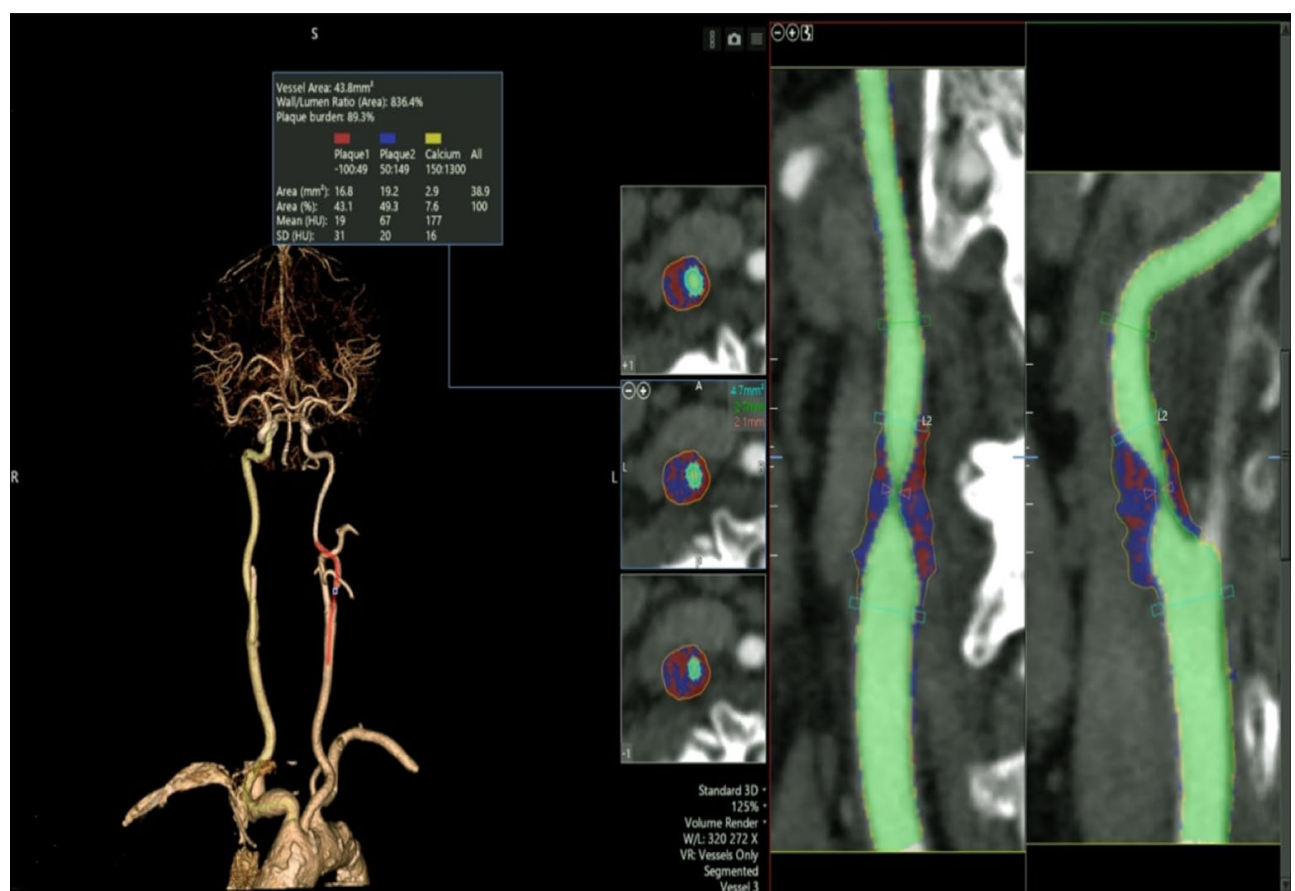

Figure 1. The images of a 54-year-old female case with the left internal carotid artery stenosis due to atheroma plaques. The post-processing images (volume rendering and curved reformat) show the plaque components with the Hounsfield Unit values and the aortic arch structures. 
The degree of carotid artery stenosis was measured according to the North American Symptomatic Carotid Endarterectomy Trial (NASCET) criteria. ${ }^{[11]}$ The plaque length was defined as the distance from the proximal to the distal of the plaque. Plaque surface morphology was as a regular or irregular surface. The total volume of plaques and the percentages of plaque components in total volume was automatically calculated according to Hounsfield unit (HU) using the SurePlaque $^{\mathrm{TM}}$ software (Toshiba Medical Systems Co., Tokyo, Japan). ${ }^{[8]}$ Plaque components were classified as lipid (-100-49 HU), fibrous (50-149 HU), and calcific (150-1300 HU) (Figure 1). ${ }^{[7]}$

\section{CAS procedure}

The CAS was administered to symptomatic patients (having an ischemic stroke or transient ischemic attack within the last six months) and angiographically found to have $>50 \%$ stenosis in the carotid artery or asymptomatic and $>70 \%$ stenosis in the carotid artery. All patients were pretreated with a combination of acetylsalicylic acid (100 mg/day) and clopidogrel (75 $\mathrm{mg}$ /day) for at least seven days before CAS. During the procedure, intravenous heparin was administered to maintain an activated clotting time of whole blood of 250 to $300 \mathrm{sec}$. Following local anesthesia administration to the inguinal region, the carotid artery was selectively catheterized with a $6 \mathrm{~F}, 90-\mathrm{cm}$ catheter sheath. After an embolic protection device (EPD) (FilterWire EZ ${ }^{\mathrm{TM}}$; Boston Scientific Inc., CA, USA) was placed, the internal carotid artery, open-cell stents (PROTÉGÉ ${ }^{\mathrm{TM}} \mathrm{RX}$; eV3 Covidien, CA, USA) was implanted on the carotid artery. Balloon dilatation was performed for all patients after stenting. Once significant bradycardia was noted during the dilatation, the required amount of atropine was injected. Finally, an angiogram of the carotid artery and intracranial circulation were performed to rule out embolic complications. The same experienced neuro-interventional radiologist performed all CAS procedures. All patients were followed in the neurocritical care unit for $24 \mathrm{~h}$ after the CAS procedure. The modified Rankin scale (mRS) was used for neurological examination by an experienced neurologist. ${ }^{[12]}$

\section{Magnetic resonance imaging (MRI)}

For detecting symptomatic or asymptomatic ischemic lesions, cranial MRI was performed in all patients using a $1.5 \mathrm{~T}$ device (Signa, GE Medical Systems; WI, USA) at 12 to $24 \mathrm{~h}$ after the CAS. Newly developed ischemic lesions were detected on the diffusion-weighted images (DWIs). The lesions were classified as ipsilateral or contralateral according to the distribution of the treated carotid artery. The patients were divided into two groups: Group $1(n=10)$ consisted of patients with a newly developed ipsilateral ischemic lesions, while the remaining patients with contralateral lesions were included in Group $2(\mathrm{n}=14)$.

After the CAS, the patients were prescribed a combination of acetylsalicylic acid $(100 \mathrm{mg} /$ day $)$ and clopidogrel (75 mg/day) for the first six months, followed by acetylsalicylic acid throughout their life.

\section{Statistical analysis}

Statistical analysis was performed using the TURCOSA version 1.0 software (Turcosa Analytics Solutions Ltd. Co., Kayseri, Turkey). Descriptive data were expressed in mean \pm standard deviation (SD) or median (min-max) for continuous variables and in number and percentage for categorical variables. The chi-square and Fisher's exact tests were used to compare the groups. The Student's t-test was used to compare the quantitative data. The threshold values for differentiation of the groups were obtained via the receiver operating characteristic (ROC) analysis. A $p$ value of $<0.05$ was considered statistically significant.

\begin{tabular}{|c|c|c|c|c|c|c|c|c|c|c|}
\hline \multirow[b]{3}{*}{ Characteristics } & \multicolumn{9}{|c|}{$\begin{array}{c}\text { Table } 1 \\
\text { Baseline demographic and clinical characteristics of patients }\end{array}$} & \multirow[b]{3}{*}{$p$} \\
\hline & \multicolumn{3}{|c|}{ Total $(n=24)$} & \multicolumn{3}{|c|}{ Group $1(n=10)$} & \multicolumn{3}{|c|}{ Group $2(n=14)$} & \\
\hline & $\mathrm{n}$ & $\%$ & Mean \pm SD & $\mathrm{n}$ & $\%$ & Mean \pm SD & $\mathrm{n}$ & $\%$ & Mean \pm SD & \\
\hline Age (year) & & & $65.4 \pm 7.4$ & & & $64.1 \pm 4.8$ & & & $67.2 \pm 8.1$ & 0.313 \\
\hline \multicolumn{11}{|l|}{ Sex } \\
\hline Female & 12 & 50 & & 6 & 60 & & 6 & 42.8 & & \\
\hline Cardiovascular risk factors & 10 & 41.6 & & 6 & 60 & & 4 & 28.5 & & 0.211 \\
\hline
\end{tabular}




\begin{tabular}{|c|c|c|c|c|c|}
\hline \multicolumn{6}{|c|}{$\begin{array}{c}\text { Table } 2 \\
\text { Structures of aortic arch }\end{array}$} \\
\hline & \multicolumn{2}{|c|}{ Group $1(n=10)$} & \multicolumn{2}{|c|}{ Group $2(n=14)$} & \multirow[b]{2}{*}{$p$} \\
\hline & $\mathrm{n}$ & $\%$ & $\mathrm{n}$ & $\%$ & \\
\hline \multicolumn{6}{|c|}{ Anatomical variations } \\
\hline Type 1 & 7 & 70 & 11 & 78.5 & \multirow{2}{*}{0.665} \\
\hline Type 2 & 3 & 30 & 3 & 21.4 & \\
\hline Elongation & 7 & 70 & 11 & 78.5 & 0.665 \\
\hline Presence of plaque & 8 & 80 & 5 & 35.7 & 0.047 \\
\hline
\end{tabular}

\section{RESULTS}

All CAS procedures were successfully performed, and all patients were neurologically intact (mRS 0) after the CAS. However, DWI images revealed acute SBI in $13(54.1 \%)$ patients. The anatomical distribution of the SBI lesions was as follows: ipsilateral lesions in $10(41.6 \%)$ patients and contralateral lesions in three (12.4\%) patients. According to the presence or absence of ipsilateral acute SBI, there were $10(41.6 \%)$ patients in Group 1 and 14 (58.3\%) patients in Group 2. There was no significant difference between the groups in terms of age and cardiovascular risk factors $(p=0.313$ and $\mathrm{p}=0.211$, respectively). Baseline demographic and clinical characteristics of the patients are summarized in Table 1.

Type 1 and Type 2 anatomical variations of the aortic arch were present in both groups. Type 1 aortic arch was present in seven (70\%) patients in Group 1 and 11 (78.5\%) patients in Group 2. No significant difference was detected between the groups regarding the anatomic variations of the aortic arch $(\mathrm{p}=0.665)$. Aortic arch elongation was seen in seven (79\%) patients in Group 1 and 11 (78.5\%) patients in Group 2, indicating no significant difference between the groups $(p=0.665)$. An aortic arch plaque was detected in eight (80\%) patients in Group 1 and five (35.7\%) patients in Group 2, indicating a significant difference between the groups $(p=0.047)$. Table 2 shows the features of the aortic arch of the patients.

The degree of carotid artery stenosis was $\geq 70 \%$ in five (50\%) patients in Group 1 and seven (50\%) patients in Group 2, indicating no significant difference between the groups $(p>0.05)$. The plaque surface morphology was irregular in eight (80\%) patients in Group 1 and five (35.7\%) patients in Group 2, indicating a higher rate of irregular morphology in Group 1 ( $\mathrm{p}=0.047$ ). The mean plaque length was $21.8 \pm 5.9 \mathrm{~mm}$ in Group 1 and $17.6 \pm 3.7 \mathrm{~mm}$ in Group 2. The mean plaque length of Group 1 was significantly higher than that of

\begin{tabular}{|c|c|c|c|c|c|c|c|c|c|}
\hline \multicolumn{10}{|c|}{$\begin{array}{r}\text { Table } 3 \\
\text { Plaque characteristics in carotid arteries as assessec }\end{array}$} \\
\hline 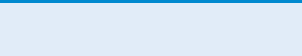 & \multicolumn{4}{|c|}{ Group $1(\mathrm{n}=10)$} & \multicolumn{4}{|c|}{ Group 2 (n=14) } & \multirow[b]{2}{*}{$p$} \\
\hline & $\mathrm{n}$ & $\%$ & Mean $\pm S D$ & UL-LL & $\mathrm{n}$ & $\%$ & Mean \pm SD & UL-LL & \\
\hline Stenosis degree $\geq 70 \%$ & 5 & 50 & & & 7 & 50 & & & 1.0 \\
\hline Irregular surface & 8 & 80 & & & 5 & 35.7 & & & 0.047 \\
\hline Length $(\mathrm{mm})$ & & & $21.8 \pm 5.9$ & $17.6-26.1$ & & & $17.6 \pm 3.7$ & $15.5-19.8$ & 0.045 \\
\hline Total volume $\left(\mathrm{mm}^{3}\right)$ & & & $991.1 \pm 279.2$ & $791-1190$ & & & $740.3 \pm 190$ & $630-850$ & 0.015 \\
\hline \multicolumn{10}{|c|}{ Percentage of components } \\
\hline Lipid component & & & $27.3 \pm 11.2$ & $11.3-35.4$ & & & $17.3 \pm 9.3$ & $11.9-22.7$ & 0.026 \\
\hline Fibrous component & & & $41.6 \pm 10.1$ & $34.3-48.9$ & & & $45.9 \pm 15.2$ & $37.1-54.8$ & 0.442 \\
\hline Calcific component & & & $30.9 \pm 19.7$ & $16.8-45.1$ & & & $36.6 \pm 21.9$ & $24.0-49.3$ & 0.519 \\
\hline
\end{tabular}




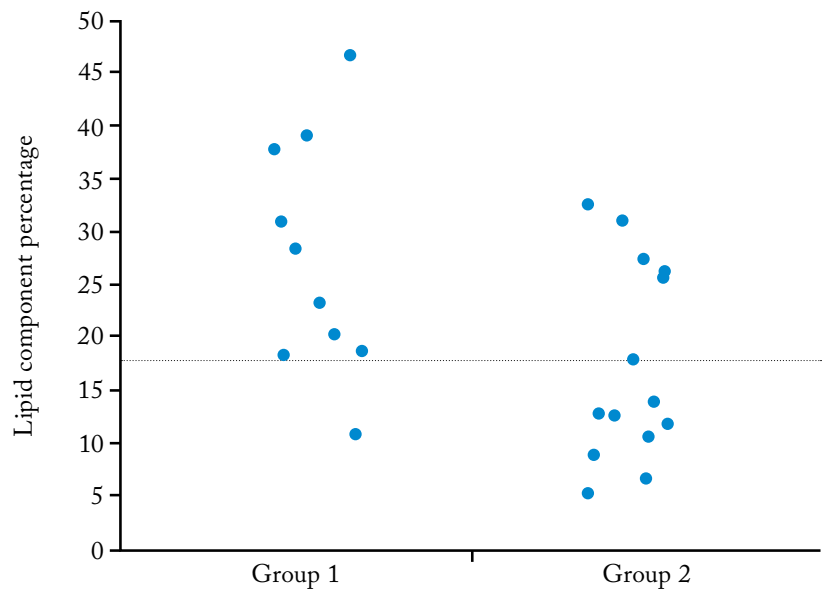

Figure 2. Distribution chart for the percentage of lipid components.

Group $2(\mathrm{p}=0.045)$. The mean total plaque volume was $991.1 \pm 279.2 \mathrm{~mm}^{3}$ in Group 1 and $740.3 \pm 190 \mathrm{~mm}^{3}$ in Group 2, indicating a significantly higher mean total plaque total volume in Group 1 ( $\mathrm{p}=0.015)$.

The percentages of plaque components in total volume were as follows: the mean percentage of lipid components was $27.3 \pm 11 \%$ in Group 1 and $17.3 \pm 9.3 \%$ in Group 2. The mean percentage of fibrous components was $41.6 \pm 10.1 \%$ in Group 1 and $45.9 \pm 15.2$ in Group 2. The mean percentage of calcific components was $30.9 \pm 19.7 \%$ in Group 1 and $36.6 \pm 21.9$ in Group 2. The comparison of the percentages of plaque components in total volume showed that the percentage of lipid components of Group 1 was higher than that of Group 2 ( $p=0.026)$. However, no significant difference was observed between the groups in terms of the percentages of fibrous and calcific components $(p=0.442$ and $p=0.519)$. The plaque characteristics in carotid arteries are shown in Table 3.

In the ROC analysis, an optimal cut-off value of percentages of lipid component in a total volume of $17.8 \%$ had a sensitivity of $64.3 \%$ and a specificity of 90\% for the prediction of ipsilateral SBI after CAS with an area under the curve (AUC) of 0.75 (Figure 2).

\section{DISCUSSION}

Carotid artery stenting is an alternative treatment method to CEA for carotid artery stenosis. However, it has a serious disadvantage due to the high incidence of SBI after the procedure. ${ }^{[13,14]}$ Silent brain infarction has adverse effects on the patients' cognitive functions in the long-term; therefore, it is important to identify patients with a high risk of SBI during CAS planning. ${ }^{[15]}$ In this study, the patients were evaluated with MDCT during CAS planning. A significant difference was found in the aortic arch structure and plaque characteristics of the carotid artery between the patients with and without SBI after CAS.

In a study, Kastrup et al. ${ }^{[16]}$ reported ipsilateral SBI after CAS with and without EPD as $49 \%$ and $67 \%$, respectively. In this study, EPD was used for all procedures, and the ratio of ipsilateral SBI after CAS was similar to Kastrup et al. ${ }^{[16]}$ Acute SBI was determined in 13 (54.1\%) patients and, according to treated carotid artery distribution, ipsilateral SBI was observed in $10(41.6 \%)$ patients and contralateral SBI was observed in three (12.5\%) patients.

In another study, Wyers et al. ${ }^{[17]}$ detected that anatomical variation or elongation of the aortic arch negatively affected the technical success and increased the risk of SBI. In this study, the presence of plaque in the aortic arch was significantly more frequent in the patients who developed SBI after CAS ( $p=0.047)$. However, there was no significant difference between the groups in terms of anatomical variation and elongation of the aortic arch. This may be due to the limited sample size of our study. Anatomical variations and degenerative changes (elongation and atherosclerosis) in the aortic arch lead to prolonged catheter manipulation time and predisposition to SBI during the procedure. ${ }^{[18]}$ Wyers et al. ${ }^{[17]}$ suggested that evaluation of aortic arch structure using MDCT for CAS planning and that patients having anatomical variation or significant elongation in the structure of aortic arch should be referred to other treatments.

Furthermore, Krapf et al. ${ }^{[19]}$ investigated the effects of carotid artery plaque length and the degree of stenosis on the development of SBI after CAS. A significantly high correlation was found between the plaque length and development of acute SBI; however, no significant correlation was observed between the degree of stenosis and the development of SBI. In the present study, carotid artery plaque length was significantly higher in the group in which ipsilateral SBI developed after CAS $(p=0.045)$. The contact between the long plaque and the catheter used in the procedure is greater than the short plaque, which increases the risk of ruptured plaque capsule and distal embolism of its components. ${ }^{[19]}$ 
In their study, Rosenkranz et al. ${ }^{[20]}$ evaluating the effect of surface morphology of carotid artery plaque on the development of SBI after CAS measured the number of microembolism during CAS by dual-frequency Doppler ultrasound. The number of microembolism was higher in plaques with an irregular surface than other plaques $(p=0.030)$. Our study revealed that the surface morphology of carotid artery plaque in Group 1 was significantly more frequently irregular, compared to Group $2(\mathrm{p}=0.047)$. The irregular surface morphology is a sign of the defect in the plaque capsule. The plaques are more vulnerable due to high blood levels of pro-inflammatory markers. Therefore, catheter contact more often causes distal embolism of plaque components. ${ }^{[21]}$

Moreover, Matsumoto et al. ${ }^{[22]}$ found that the amount of development SBI during CAS was positively correlated with the total volume of plaque and proportion of lipid component in total volume $(\mathrm{r}=0.480, \mathrm{p}=0.015$ and $\mathrm{r}=0.561, \mathrm{p}=0.001$, respectively). Uchiyama et al. ${ }^{[23]}$ found that lipid componentweighted plaques, with a mean $\mathrm{HU}$ value of $<0$, were found to be more prone to the development of SBI after CAS. In the present study, the total volume of plaque and percentage of lipid component was significantly higher in patients who developed SBI than the others $(p=0.015$ and $p=0.026$, respectively). For differentiation of the patients with and without SBI after CAS, the percentage of lipid component in total volume of $>17.8 \%$ was found to be a selectivity cut-off value. As the total volume of plaque and proportion of lipid component in total volume increase, plaque resistance to mechanical stress decreases and a predisposition to microembolism occurs due to the catheter contact. ${ }^{[24]}$

In the current study, we found no significant differences in the percentages of calcific components between the groups $(p=0.519)$. Rather than the percentage of the calcific components, the localization of the calcific components appears to be the factor affecting the development of SBI. The calcific component can be located in the blood vessel wall in two different locations: tunica intima and tunica media/adventitia. Peripheral calcific plaques located on tunica media/adventitia are larger and less elastic and, therefore, the risk of development SBI is higher in such plaques. ${ }^{[25]}$

Nonetheless, there are several limitations to this study. First, this study has a retrospective design with a small sample size which may have affected the statistical results. Unfortunately, most of the patients who underwent CAS in our hospital were advanced age and prone to contrast nephropathy and, therefore, the number of patients evaluated using MDCT was limited. Second, we did not include intraplaque hemorrhage in plaque component classification. Intraplaque hemorrhage cause plaque instability and predisposition to SBI. Third, the study included only a single observer, inter-observer agreement was unable to be measured.

In conclusion, in CAS planning, it is of utmost importance to identify patients at high risk of developing SBI after CAS. Structures of the aortic arch and characteristics of carotid artery plaque may be useful markers to predict the risk of development of SBI. Post-processing analysis of high-spatial resolution images obtained using MDCT may be helpful to determine these properties successfully.

\section{Declaration of conflicting interests}

The authors declared no conflicts of interest with respect to the authorship and/or publication of this article.

\section{Funding}

The authors received no financial support for the research and/or authorship of this article.

\section{REFERENCES}

1. Kış M, Merdanoğlu M, Balkanay M. A comparison of modified eversion endarterectomy versus classical endarterectomy in the surgical treatment of carotid artery stenosis. Cardiovascular Surgery and Interventions 2020;7:163-9.

2. Luebke T, Brunkwall J. Carotid artery stenting versus carotid endarterectomy: Updated meta-analysis, metaregression and trial sequential analysis of short-term and intermediate-to long-term outcomes of randomized trials. J Cardiovasc Surg (Torino) 2016;57:519-39.

3. Crawley F, Clifton A, Buckenham T, Loosemore T, Taylor RS, Brown MM. Comparison of hemodynamic cerebral ischemia and microembolic signals detected during carotid endarterectomy and carotid angioplasty. Stroke 1997;28:2460-4.

4. Maggio P, Altamura C, Landi D, Migliore S, Lupoi D, Moffa F, et al. Diffusion-weighted lesions after carotid artery stenting are associated with cognitive impairment. J Neurol Sci 2013;328:58-63.

5. Bijuklic K, Wandler A, Varnakov Y, Tuebler T, Schofer J. Risk factors for cerebral embolization after carotid artery stenting with embolic protection: A diffusion-weighted magnetic resonance imaging study in 837 consecutive patients. Circ Cardiovasc Interv 2013;6:311-6. 
6. Yamada K, Yoshimura S, Kawasaki M, Enomoto Y, Takano $\mathrm{K}$, Asano T, et al. Prediction of silent ischemic lesions after carotid artery stenting using virtual histology intravascular ultrasound. Cerebrovasc Dis 2011;32:106-13.

7. Varrassi M, Sferra R, Gravina GL, Pompili S, Fidanza RC, Ventura $\mathrm{M}$, et al. Carotid artery plaque characterization with a wide-detector computed tomography using a dedicated post-processing 3D analysis: Comparison with histology. Radiol Med 2019;124:795-803.

8. Zheng J, Chang Z, Hou Y, Liu Z, Lu Z, Guo Q, et al. Accuracy of MDCT for detection and identification of carotid atherosclerotic plaque in a rabbit model. AJR Am J Roentgenol 2014;202:W176-81.

9. Celikyay ZR, Koner AE, Celikyay F, Denız C, Acu $B$, Firat MM. Frequency and imaging findings of variations in human aortic arch anatomy based on multidetector computed tomography data. Clin Imaging 2013;37:1011-9.

10. Sugawara J, Hayashi K, Yokoi T, Tanaka H. Age-associated elongation of the ascending aorta in adults. JACC Cardiovasc Imaging 2008;1:739-48.

11. Ferguson GG, Eliasziw M, Barr HW, Clagett GP, Barnes RW, Wallace MC, et al. The North American Symptomatic Carotid Endarterectomy Trial: Surgical results in 1415 patients. Stroke 1999;30:1751-8.

12. Bonita R, Beaglehole R. Recovery of motor function after stroke. Stroke 1988;19:1497-500.

13. Bonati LH, Jongen LM, Haller S, Flach HZ, Dobson J, Nederkoorn PJ, et al. New ischaemic brain lesions on MRI after stenting or endarterectomy for symptomatic carotid stenosis: A substudy of the International Carotid Stenting Study (ICSS). Lancet Neurol 2010;9:353-62.

14. Piñero P, González A, Mayol A, Martínez E, GonzálezMarcos JR, Boza F, et al. Silent ischemia after neuroprotected percutaneous carotid stenting: A diffusion-weighted MRI study. AJNR Am J Neuroradiol 2006;27:1338-45.

15. Setacci C, Chisci E, Setacci F, Iacoponi F, de Donato G, Rossi A. Siena carotid artery stenting score: A risk modelling study for individual patients. Stroke 2010;41:1259-65.
16. Kastrup A, Nägele T, Gröschel K, Schmidt F, Vogler E, Schulz J, et al. Incidence of new brain lesions after carotid stenting with and without cerebral protection. Stroke 2006;37:2312-6.

17. Wyers MC, Powell RJ, Fillinger MF, Nolan BW, Cronenwett JL. The value of $3 \mathrm{D}-\mathrm{CT}$ angiographic assessment prior to carotid stenting. J Vasc Surg 2009;49:614-22.

18. Lin SC, Trocciola SM, Rhee J, Dayal R, Chaer R, Morrissey $\mathrm{NJ}$, et al. Analysis of anatomic factors and age in patients undergoing carotid angioplasty and stenting. Ann Vasc Surg 2005;19:798-804.

19. Krapf H, Nägele T, Kastrup A, Bühring U, Grönewäller E, Skalej M, et al. Risk factors for periprocedural complications in carotid artery stenting without filter protection: A serial diffusion-weighted MRI study. J Neurol 2006;253:364-71.

20. Rosenkranz M, Russjan A, Goebell E, Havemeister S, Thomalla G, Cheng B, et al. Carotid plaque surface irregularity predicts cerebral embolism during carotid artery stenting. Cerebrovasc Dis 2011;32:163-9.

21. Prabhakaran S, Rundek T, Ramas R, Elkind MS, Paik MC, Boden-Albala B, et al. Carotid plaque surface irregularity predicts ischemic stroke: The northern Manhattan study. Stroke 2006;37:2696-701.

22. Matsumoto S, Nakahara I, Higashi T, Iwamuro Y, Watanabe Y, Takezawa M, et al. Fibro-fatty volume of culprit lesions in Virtual Histology intravascular ultrasound is associated with the amount of debris during carotid artery stenting. Cerebrovasc Dis 2010;29:468-75.

23. Uchiyama N, Misaki K, Mohri M, Watanabe T, Hirota $\mathrm{Y}$, Nakada $\mathrm{M}$, et al. Association between carotid plaque composition assessed by multidetector computed tomography and cerebral embolism after carotid stenting. Neuroradiology 2012;54:487-93.

24. Shah PK. Mechanisms of plaque vulnerability and rupture. J Am Coll Cardiol 2003;41(4 Suppl S):15S-22S.

25. Barrett HE, Cunnane EM, Kavanagh EG, Walsh MT. On the effect of calcification volume and configuration on the mechanical behaviour of carotid plaque tissue. J Mech Behav Biomed Mater 2016;56:45-56. 